Original Research Paper

\title{
An Efficient Denoising Algorithm for Impulse Noise Removal
}

\author{
${ }^{1}$ A. Rajamani and ${ }^{2}$ V. Krishnaveni \\ ${ }^{1}$ Department of Electronics and Communication Engineering, PSG Polytechnic College, Coimbatore, India \\ ${ }^{2}$ Department of Electronics and Communication Engineering, PSG College of Technology, Coimbatore, India
}

Article history

Received: 18-03-2014

Revised: $10-05-2014$

Accepted: 25-07-2014

Corresponding Author: A. Rajamani

Department of Electronics and Communication Engineering, PSG Polytechnic College, Coimbatore, India

Email: rajamani_saranya@rediffmail.com

\begin{abstract}
Now-a-days the images acquired by the digital cameras and defective sensors tend to introduce noises during either image acquisition or transmission process. The quality of the image is degraded in a significant measure. Lot of research works was carried out for several decades to denoise the impulse noise and each approach has its own merits and demerits. This study deals with a new denoising approach for the gray scale images to discard fixed type salt and pepper noise present in the images. This algorithm was implemented for gray scale images such as Lena and cameraman and the performance results are really challenging both qualitative and quantitative wise. This study considered the performance metrics like PSNR and MSE for quantitative measure and presents better results for low density noise level to high density noise level (up to 100\%), when compared to other existing filters. The visual interpretation shows that this method proves better in qualitative analysis by human perception too. In addition to this the proposed approach decreases the computational and hardware complexity by an appreciable manner since traditional sorting schemes does many comparisons and that were very much avoided. Thus very fast operation could be achieved. This study deals with neighborhood pixel comparison which are confined to previous pixel and the pixel next to the processing pixel under consideration, the absence of sorting saves much time and number of operations, which in turn speed of operation is increased and better reconstruction of images is achieved.
\end{abstract}

Keywords: Salt and Pepper Noise, Standard Median Filter, Peak Signal to Noise Ratio, Mean Square Error

\section{Introduction}

The digital images which are taken by a camera system yields noises during image acquisition or transmission, due to the reasons such as the out of focus of the picture due to motion of camera, troublesome weather, atmospheric turbulence, sensor problem, storage of information and noise during digital conversion process such as sampling and quantization. But one of the major hindrances to the widespread use of real time images in real world applications is the noise which gets automatically added to them during either being capturing images or being transmitting the bits. Thus it is mandatory to remove those noises which make it difficult to interpret the image for further processing. There are various types of noises that will affect an image's quality namely Gaussian noise, salt and pepper noise, random noise (Dong and $\mathrm{Xu}, 2007$ ), speckle noise, to name a few. Each type of noise is defined by its own characteristic features and removal of these noise components from the image is performed by various ways. Some of the noises due to transmission are mainly affected by (salt and pepper) which is additive in nature. The additive noise is systematic and easily modeled than the multiplicative noise. Thus denoising is essential for visually pleasant images by improving image quality and also will be helpful for other postprocessing operations like segmentation and registration.

It is well known that the picture elements in an image are considered as noise (Vijaykumar et al., 
2008) when there is an abrupt change or major difference in pixel values between the neighbor pixels. This abrupt change like a spike, in pixel values constitutes the salt (pixel value 255) and pepper (pixel value 0 ) noise in an image. In an image it is viewed as black and white dots. The source of this kind of interference is mainly due to bit error in transmission of signals and also due to flecks of dust inside the camera device and over heated or malfunctioning Charge Coupled Devices (CCDs). Mostly many algorithms which are indebted for converting the image sensor information to a visual image, whether it might be in camera or on a computer, which is very much prone to some kind of noise reduction. There are so many procedures for this to happen, but all attempt to verify whether the actual differences in pixel values constitute noise or real photographic information and does the mean for the former while trying to preserve the latter. However, all the approaches exhibits a tradeoff made between noise removal and preservation of fine, low-contrast detail that may have characteristic similar to noise. There are two types of impulse noises (Thirumurugan et al., 2014) namely random valued impulse noise and fixed valued impulse noise. The random valued noise (Shaik, 2012) will take any value in between 0 to 255 and can be interpreted either as information or as noise. Whereas the fixed noise will have either 0 or 255. This study made an attempt to remove salt and pepper fixed noise from Gray scale images effectively. The noise model (Jasdeep and Garg, 2013) for the fixed salt and pepper noise is given as:

$$
\begin{aligned}
& Y(i, j)=255 \text { with a probability of } \frac{P}{2} \\
& =0 \text { with a probability of } \frac{P}{2} \\
& X(i, j) \text { with a probability of } 1-P
\end{aligned}
$$

The $50 \%$ probability for each type of fixed noise and remaining pixels are considered as non noisy.

The quick review of some existing filters is described as follows.

Since the linear filters are not preserving the edges well (Roomi et al., 2010) and have the problem of blurring effect, the non linear filter known as Standard Median Filter (SMF) technique was performed, which is very useful in preserving the sharp edges of an image (Raymond et al., 2005) and is characterized by the high frequency details (Raymond et al., 2005) present in it, while filtering noise. It removes the salt (255) and pepper (0) noise from images efficiently without any blur in its output. When the difference between a processing pixel value and its neighborhood pixels is large, it is characterized as noise and can be replaced by the median value after all the pixels in the window are sorted in the ascending or descending order. The detailed step for performing median filtering is as follows:

- Take an input array of $\mathrm{N}$ values. ( $\mathrm{N}$ represents the size of the window pixels) for a sliding window of size $m x n$

- $\quad$ Sort the input array in the ascending order $P_{i} 1, j 1<$ $P i 2, j 2 \ldots \ldots<P i N, P j N$

- Find the median value and replace the centre pixel by the median value

- Perform it for the entire image by sliding the window to the both horizontal and vertical directions

- Repeat the above said process by taking a new set of $\mathrm{N}$ values

The limitation of this approach (Shuqun and Karim, 2002) is obvious that it will alter non noisy pixels also, since uniform treatment for all pixel candidates and results high computational complexity even though it possesses good edge preservation.

The other median based filters (Srinivasan and Ebenezer, 2007) like Adaptive Median Filter (AMF) (Hwang and Hadded, 1995), Switching Median Filter (PSMF) are good only for low density noise but poor for high density noise (Aiswarya, et al., 2010).

To deal with high density noise the algorithms such as Decision Based Algorithm (DBA) and Decision based Unsymmetric Trimmed Median Filter (DBUTMF) and Modified Decision Based Unsymmetric Trimmed Median Filter (MDBUTMF) are proposed (Esakkirajan et al., 2011) and all are not performing well for high noise density of about $70 \%$ and above and blurring effect takes place.

This study effectively deals with the limitations of the above said problems and tries to overcome by introducing an efficient algorithm in which the noisy pixels is replaced by the neighborhood pixel only when the neighbor is not a noisy pixel and it is analyzing all neighbor pixels for better pixel replacement. This algorithm provides challenging results when compared to other denoising approaches in terms of higher Peak Signal to Noise Ratio (PSNR) and lower Mean Square Error (MSE).

The paper is organized as the first section briefs the introduction and describes the other competitive filters which are taken for comparison with the proposed algorithm. The flowchart and the detailed steps of proposed algorithm are given in the second 
section. The discussion about the indicator for performance measure metrics are given in third section. The forth section briefs inferences of results and finally fifth section gives conclusion of the tasks carried out in this study.

\section{Proposed Algorithm}

The Gray scale Lena and cameraman images are taken and its characteristic behavior and noise models are analyzed for the effective proposal of this noise removal technique. Based on the nature of noise and its behavior inferred from the image, an efficient algorithm is being introduced, which is used to remove noise density upto $90 \%$ and even for higher noise densities greater than $90 \%$. This proposed algorithm better suits for all noise density level right from 10 to $100 \%$.

Initially the original image is acquired and added with some percentage $(10 \%)$ of noise and every time noise level is incremented with a step size of $10 \%$ and goes up to $100 \%$ noise density.

In the traditional sorting approach, many number of comparisons, computational operations (Shuqun and Karim, 2002) are done and moreover the same process is to be repeated for all the pixels irrespective of whether it is noisy or not. Also it will alter the non noisy pixel values and the entire pixels involved in the sorting process. It unnecessarily performs for non noisy pixels too, which in turn results in high computational and hardware complexity. Moreover speed of operation is also reduced.

To overcome all the above limitations one new approach has been proposed and implemented in Matlab tool. The proposed approach outperforms and some promising results were obtained and thus proves its excellence by giving much improved PSNR, decrease in MSE error than traditional competitive algorithms.

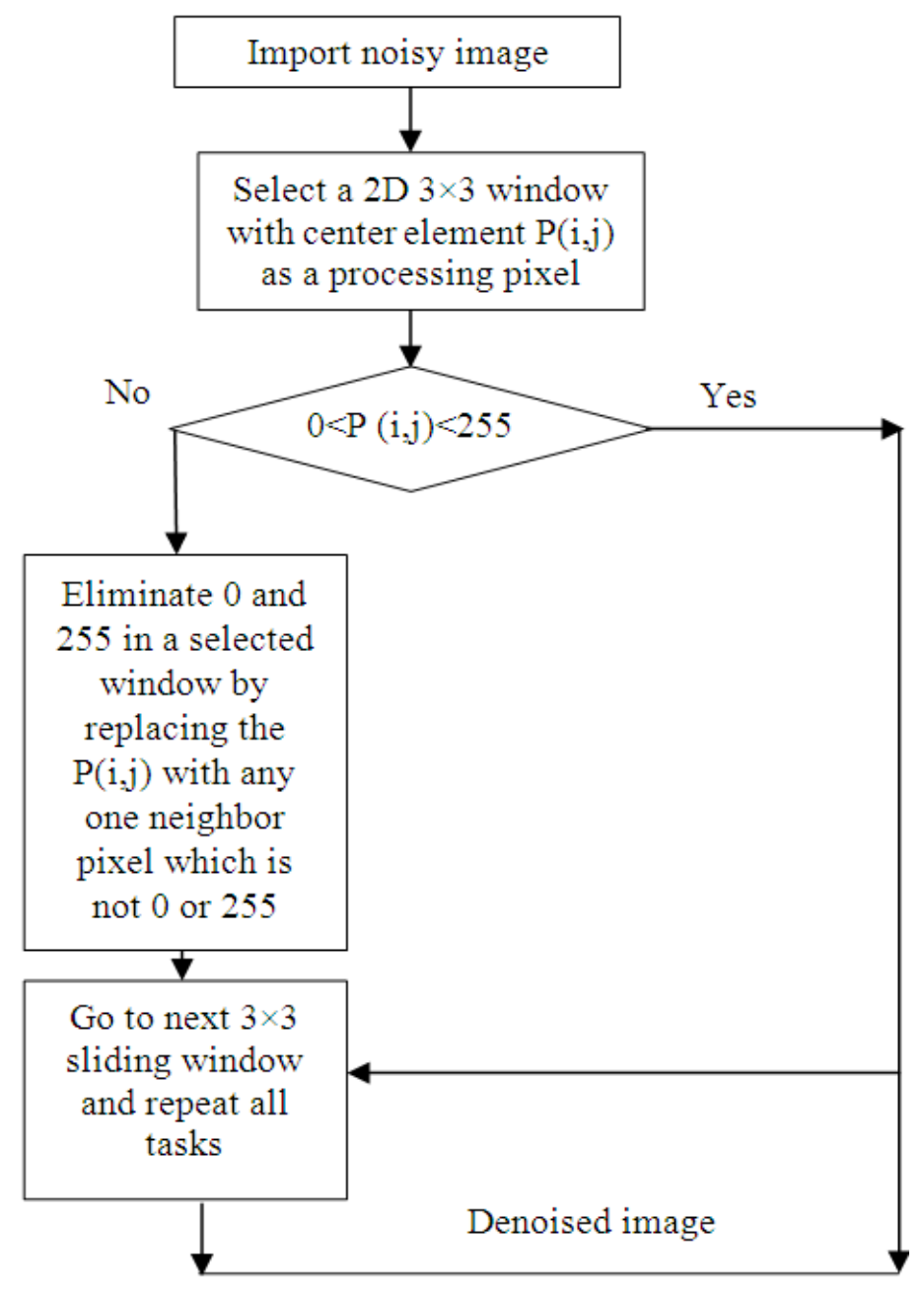

Fig. 1. Flowchart of the proposed algorithm 
The steps of the proposed filter are described as follows and Fig. 1 presents the detailed Flowchart of the proposed algorithm. Proposed Algorithm:

Step 1 = Select a two dimensional moving window with a window of size $3 \times 3$ as sub matrix among the entire image Assume that the centre pixel being processed is $P(i, j)$

Step $2=$ Check the $P(i, j)$ for the condition, $0=P(i, j)=$ 255 if yes then $P(i, j)$ is not a noisy pixel and its value is left intact

Step $3=$ If $0 P(i, j)=255$, then $P(i, j)$ is a noisy pixel

Step4 = The centre pixel $P(i, j)$ under consideration is to be replaced by the previous neighborhood value i.e., $P(i-1, j)$ then two cases are possible as given in Case i) and ii)

Case $\mathrm{i})=$ If the selected window contains $P(\mathrm{i}-1, \mathrm{j})$ and is not equal to 0 or 255 , then replace $P(i, j)$ with the $\mathrm{P}(\mathrm{i}-1, \mathrm{j})$ value

Case ii $)=$ If the selected window contains $P(i-1, j)$ and is equal to 0 or 255 , then replace $P(i, j)$ with post neighborhood pixel of $P(i, j)$, i.e., replace with $P(i-1, j)$ value

Step $5=$ In case both previous and post neighborhood pixels are again either equal to 0 or 255 , then replace $P(i, j)$ with $P(i, j+1) P(i, j-1)$ value

Step $6=$ Next sliding window is selected and processed as given above

Step $7=$ Repeat steps 1 to 5 until all the pixels in the entire image are processed

\section{Quantitative Analysis}

The image reconstruction quality could be assessed by the metrics like PSNR and MSE. This study exploits the metrics in order to determine the performance of the noise removal algorithm and the following metric parameters are analyzed:

- Mean Square Error (MSE)

- Peak Signal-to-Noise Ratio (PSNR)

The metric results of these parameters are analyzed and based on the inferences drawn from them the performance of the proposed algorithm is estimated.

\section{Mean Square Error}

The MSE is the cumulative error between the processed and the original measures the average of the squares of the "errors". The error is the amount of measure by which the value implied by the estimator differs from the quantity to be estimated according to the Equation 3. The difference occurs because of randomness where:

$$
M S E=\frac{1}{m n} \sum_{i=0}^{m-1} \sum_{j=0}^{n-1}[I(i, j)-K(i, j)]^{2}
$$

where, $I(i, j)$ is the original image, $K(i, j)$ is the approximated version (which is actually the image with noise) $m, n$ is the dimensions of the images.

\section{Peak Signal-to-Noise Ratio}

Peak Signal-to-Noise Ratio (PSNR) is the ratio between the maximum signal power to the corrupting noise power that affects the fidelity of its representation. Since many signals have a very wide dynamic range, PSNR is generally expressed in terms of the logarithmic decibel scale which can be seen in Equation 4. The PSNR is mostly used as a measure of quality of reconstruction of the image under process. The signal is the original information and the noise is the error introduced by compression, transmission or storage of information.

For example when comparing compression codecs, it is used as a task of approximation to human perception of reconstruction of image quality. Therefore in some cases one reconstruction itself may appear to be the original than another, though it has a lower PSNR (It is highly accepted that always a higher PSNR will normally indicate that the reconstruction is of higher quality) Equation 4:

$P S N R=10 \log _{10}\left(\operatorname{Max}^{2} / M S E\right)$

In the case of large size images, the calculation of MSE and PSNR seems to be difficult, since the term $1 /(m * n)$ tends to zero in the MSE formula shown in Equation 3.

Since MSE tends to zero, naturally PSNR value tends to become infinity. Therefore, these images are to be resized to a standard size of $512 \times 512$, so that none of the vital information like important details and features of the images are altered and the interest is on their dimensions which are manipulated to determine the performance metrics. When considering small size images, no resizing is necessary. The metric parameters are directly computed and the results obtained.

\section{Results}

The proposed algorithm results are presented in terms of qualitative and quantitative wise and a comparative study was done among other algorithms such as Decision Based Algorithm (DBA) (Zhang et al., 2013).

And Decision based Unsymmetric Trimmed Median Filter (DBUTMF) and Modified Decision Based 
Unsymmetric Trimmed Median Filter (MDBUTMF) and is shown as follows.

Table1 describes the comparison of PSNR values of different algorithms for Lena image at various noise densities, Table 2 shows the comparison results of MSE values of different algorithms for Lena, image at various noise densities, Table 3 compares the results of PSNR values of different algorithms for cameraman Image and Table 4 presents the comparison results of MSE and PSNR values of Proposed Algorithm (PA) for cameraman image at various noise densities.

The proposed algorithm has been compared with the other competitive algorithms such as SMF, AMF, PSMF, WMF, TDF, LDS, DBA, MDBA, MDBUTMF and MAUTMPF. The recently developed algorithm which was proposed by the authors (Saravanakumar et al., 2014) reported that the removal of high density impulse noise using morphological based adaptive unsymmetrical trimmed mid-point filter presented the PSNR, MSE values as 28.17 and 99 at $90 \%$ noise density respectively. For the same case much improvement was shown by the proposed algorithm as 32.3569 and 37.7734. From the quantitative Analysis it is proven that the proposed algorithm yields much better results in terms of high PSNR and low MSE for all noise density level.

Figure 2 shows the visual qualitative results of the proposed algorithm for Lena and cameraman images for the noise densities from 10 to $90 \%$. The qualitative performance also claims its effectiveness of good restoration and it is outperforming than other existing filtering algorithms.

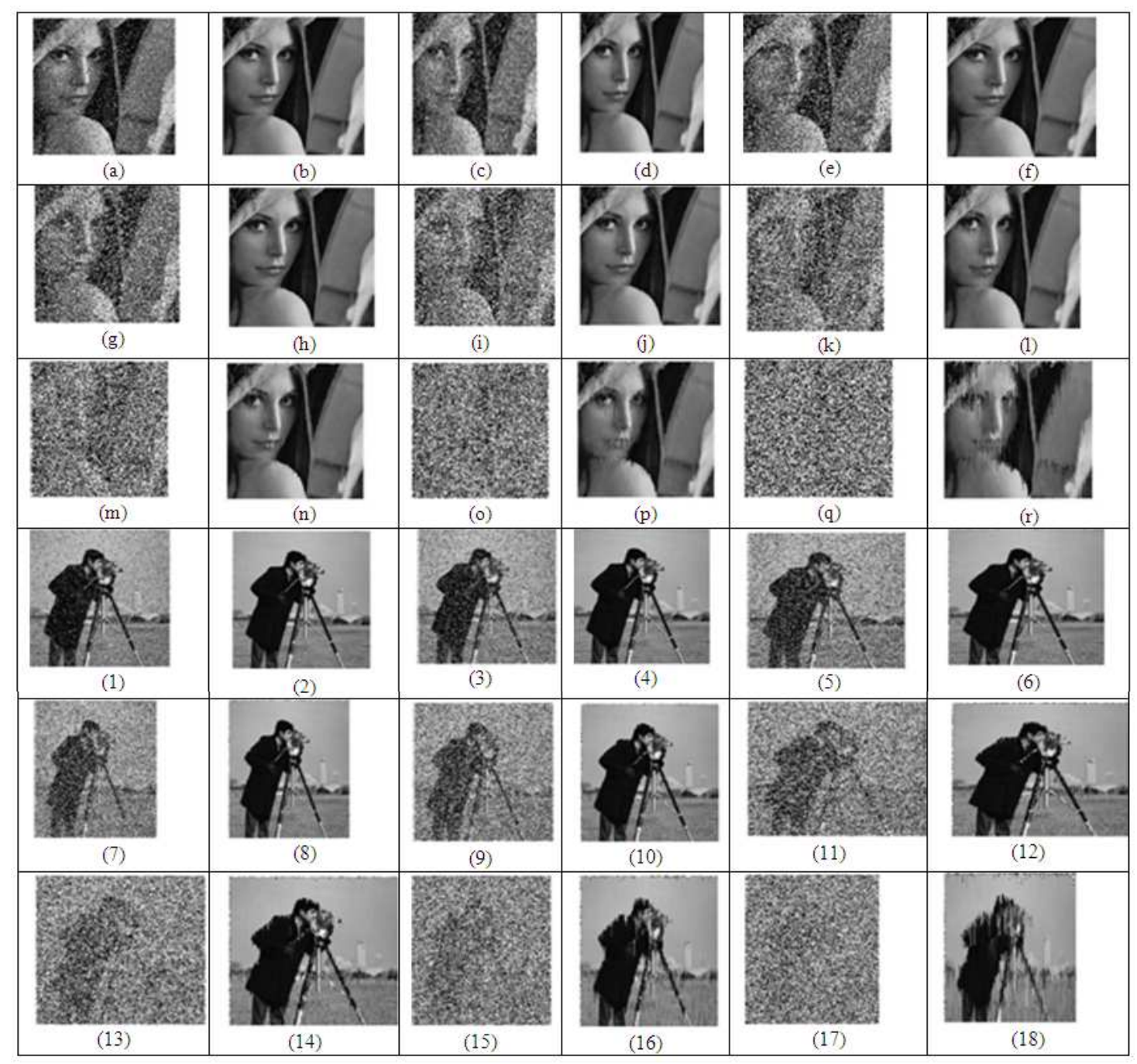

Fig. 2. Qualitative results of the proposed algorithm for Lena and cameraman images for the noise densities from 10 to $90 \%$, (a, c, e, g, i, k, m, o, q represents corrupted Lena images from 10 to $90 \%$ respectively and b, d, f, h, j, l, n, p, r represents corresponding restored images), (1,3,5,7,9,11,13,15,17 represents corrupted cameraman images from 10 to $90 \%$ respectively and $2,4,6,8,10,12,14,16,18$ represents corresponding restored images) 
Table 1. Comparison results of PSNR values of different algorithms for Lena, image at various noise densities

Noise

\begin{tabular}{|c|c|c|c|c|c|c|c|c|c|c|c|}
\hline density (\%) & SMF & $\mathrm{AMF}$ & PSMF & WMF & TDF & LDS & DBA & MDBA & MDBUTMF & MAUTMPF & PA \\
\hline 10 & 22.750 & 29.480 & 30.220 & 34.22 & 35.53 & 37.15 & 38.43 & 36.94 & 37.91 & 42.53 & 47.3012 \\
\hline 20 & 18.750 & 28.300 & 28.390 & 27.08 & 33.25 & 33.25 & 37.36 & 32.69 & 34.78 & 39.50 & 43.8785 \\
\hline 30 & 15.300 & 27.100 & 25.520 & 21.66 & 31.73 & 30.76 & 35.92 & 30.41 & 32.29 & 37.99 & 41.8121 \\
\hline 40 & 13.180 & 25.550 & 22.490 & 17.57 & 30.65 & 28.81 & 34.12 & 28.49 & 30.32 & 36.56 & 40.0267 \\
\hline 50 & 11.820 & 24.040 & 19.130 & 14.20 & 29.78 & 26.71 & 32.21 & 26.52 & 28.18 & 35.37 & 38.8210 \\
\hline 60 & 11.000 & 21.070 & 12.100 & 11.64 & 26.12 & 25.04 & 30.43 & 24.41 & 26.43 & 34.13 & 37.3508 \\
\hline 70 & 10.720 & 16.100 & 11.840 & 09.49 & 24.67 & 22.90 & 28.62 & 22.47 & 24.30 & 32.55 & 36.0519 \\
\hline 80 & 9.080 & 11.600 & 08.000 & 07.90 & 22.51 & 20.64 & 26.23 & 20.44 & 21.70 & 30.70 & 34.2873 \\
\hline 90 & 8.250 & 08.002 & 06.570 & 06.56 & 20.04 & 18.98 & 23.94 & 17.56 & 18.40 & 28.17 & 32.3589 \\
\hline
\end{tabular}

Table 2. Comparison results of MSE values of different algorithms for Lena, image at various noise densities

\begin{tabular}{|c|c|c|c|c|c|c|c|c|c|c|c|}
\hline $\begin{array}{l}\text { Noise } \\
\text { density (\%) }\end{array}$ & $\mathrm{SMF}$ & AMF & PSMF & WMF & TDF & LDS & DBA & MDBA & MDBUTMF & MAUTMPF & PA \\
\hline 10 & 25.90 & 33.76 & 24.90 & 20.34 & 23.54 & 12.62 & 20.64 & 9.300 & 4.56 & 3.63 & 1.2105 \\
\hline 20 & 46.10 & 36.60 & 37.60 & 56.25 & 57.45 & 30.95 & 38.56 & 18.600 & 17.12 & 7.29 & 2.6621 \\
\hline 30 & 117.50 & 83.53 & 43.40 & 179.56 & 184.49 & 54.98 & 56.10 & 29.100 & 26.34 & 10.33 & 4.2842 \\
\hline 40 & 305.20 & 125.66 & 203.45 & 444.36 & 456.74 & 86.10 & 81.36 & 35.400 & 30.40 & 14.35 & 6.4627 \\
\hline 50 & 677.04 & 147.34 & 352.14 & 895.50 & 898.67 & 139.67 & 113.12 & 41.580 & 41.23 & 18.87 & 8.5307 \\
\hline 60 & 1330.06 & 254.77 & 478.85 & 1586.42 & 1570.62 & 204.92 & 163.84 & 130.700 & 61.25 & 25.13 & 11.9673 \\
\hline 70 & 2241.07 & 466.56 & 789.23 & 2524.05 & 2657.54 & 335.50 & 251.85 & 99.960 & 71.50 & 36.15 & 16.1395 \\
\hline 80 & 3464.50 & 517.56 & 2205.32 & 3672.36 & 3679.68 & 565.65 & 305.39 & 169.610 & 118.90 & 55.36 & 24.2296 \\
\hline 90 & 4883.21 & 1041.99 & 3987.35 & 5031.06 & 5132.98 & 830.55 & 730.72 & 240.925 & 134.00 & 99.00 & 37.7734 \\
\hline
\end{tabular}

Table 3. Comparison results of PSNR values of different algorithms for cameraman image

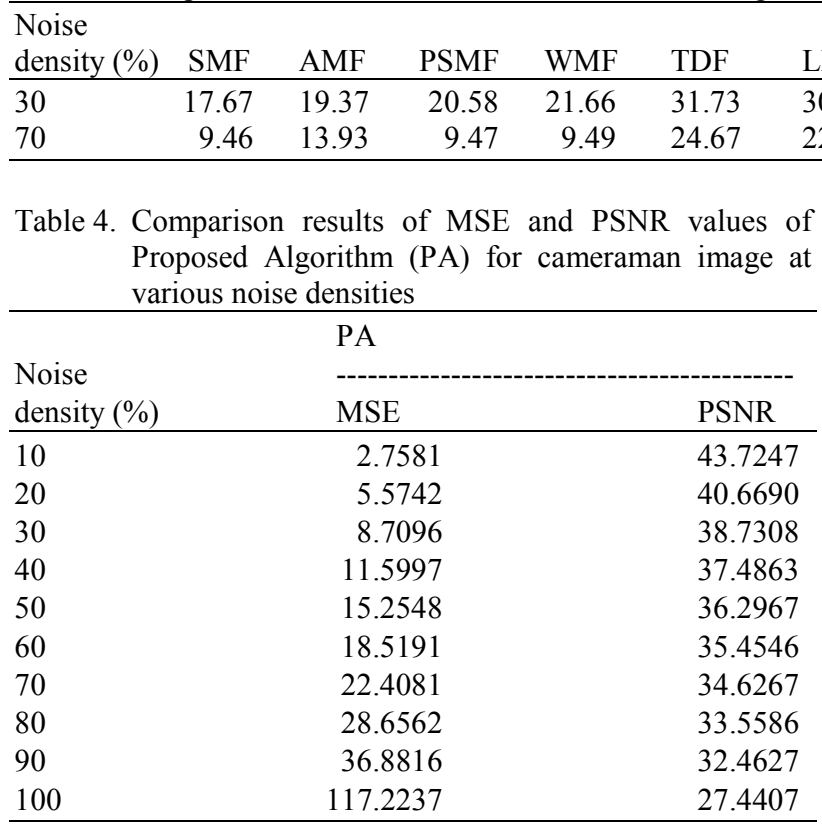

\section{Discussion}

The proposed algorithm has been successfully tested for different noise densities for Gray scale Lena and cameraman images. The performance of the proposed algorithm is tested for all levels of noise corruption (up to $100 \%$ ) with an increment size of $10 \%$. The proposed algorithm proves its promising results by much improved PSNR value and decrease

in MSE value than other competitive algorithms. Usually all algorithms perform well for low level noise densities and exhibit blur for high level noise density. But this algorithm yields better result with a high PSNR value of 32.3589 at $90 \%$ noise density and less MSE value of 37.7734 , compared to other competitive filters.

\section{Conclusion}

In this study a new efficient denoising algorithm for the effective removal of impulse noise is presented and the algorithm was compared with earlier non linear median based filtering approaches. This proposed algorithm proved that it is performing well for all the noise density level in terms of quantitative metric wise as higher PSNR and lesser MSE. It has been shown that the performance of the algorithm for the better visual interpretation in qualitative wise also. The performance is tested with lena and cameraman gray scale images for various noise densities. Further this study might be extended for color images and video sequences. The limitation of this approach is that it is more suitable for still images. Extension to this algorithm may be carried out for random valued impulse noise, since the 
detection of random valued noise is really challenging one. The Future research might be concentrated by increasing the metric PSNR and decreasing the metric MSE, with not compromising the computational complexity and speed of operation.

\section{Author's Contributions}

All authors equally contributed in this work.

\section{Ethics}

This article is original and contains unpublished material. The corresponding author confirms that all of the other authors have read and approved the manuscript and no ethical issues involved.

\section{References}

Aiswarya, K., V. Jayaraj and D. Ebenezer, 2010. A new and efficient algorithm for the removal of high density salt and pepper noise in images and videos. Proceedings of the 2nd International Conference on Computer Modeling and Simulation, Jan. 22-24, IEEE Xplore Press, Sanya, Hainan, pp: 409-413. DOI: $10.1109 /$ ICCMS.2010.310

Dong, Y. and $\mathrm{S}$. $\mathrm{Xu}, 2007$. A new directional weighted median filter for removal of random valued impulse noise. IEEE Signal Process. Lett., 14: 193-196. DOI: 10.1109/LSP.2006.884014

Esakkirajan, S., T. Veerakumar, A.N. Subramanyam and C.H.P. Chand, 2011. Removal of high density salt and pepper noise through modified decision based unsymmetric trimmed median filter. IEEE Signal Process. Lett., 5: 287-290. DOI: $10.1109 /$ LSP.2011.2122333

Hwang, H. and R.A. Hadded, 1995. Adaptive median filter: New algorithms and results. IEEE Trans. Image Proc., 4: 499-502. DOI: 10.1109/83.370679

Jasdeep, K and M. Garg, 2013. An improved weighted median filter for the image processing application. 43: 45-347.
Raymond, H., C. Chung-wa and M. Nikolova, 2005. Salt and pepper noise removal by median type noise detectors and detail-preserving regulari zation, IEEE trans. Image Process., 14: 1479-1485.

Roomi, S.S.M., P.L.M. Karuppi, P. Rajesh and B.G. Revathi, 2010. A particle swarm optimization based edge preserving impulse noise filter. J. Comput. Sci., 6: 1014-1020.

Saravanakumar, S., A. Ebenezer Jeyakumar and K.N. Vijeyakumar, 2014, Removal of high density impulse noise using morphological based adaptive unsymmetrical trimmed mid-point filter. J. Comput. Sci., 10: 1307-1314. DOI: 10.3844/jcssp.2014

Shaik, A.I., 2012. Impulse noise detection and filtering based on adaptive weighted median filter. Res. Invent: Int. J. Eng. Sci., 11: 49-54.

Shuqun, Z. and M.A. Karim, 2002. A new impulse detector for switching median filters. IEEE Sig. Process. Lett., 9: 360-363. DOI: 10.1109/LSP.2002.805310

Srinivasan, K.S. and D. Ebenezer, 2007. A new fast and efficient decision-based algorithm for removal of High-density impulse noises. IEEE Sig. Process. Lett., 3: 189-192. DOI: 10.1109/LSP.2006.884018

Thirumurugan, P., S. Sasikumar and C. Sugapriya, 2014. FPGA implementation and analysis of impulse noise reduction in images. Am. J. Applied Sci., 11: 1041-1048. DOI: $10.3844 /$ ajassp.2014.1041.1048

Vijaykumar, V.R., P.T. Vanathi, P. Kanagasabapathy and D. Ebenezer, 2008. High density impulse noise removal using robust estimation based filter. IAENG Int. J. Comput. Sci., 3: 1-10.

Zhang, X., Y. Zhan, M. Ding, W. Hou and Z. Yin, 2013. Decision-based non-local means filter for removing impulse noise from digital images. Sig. Process., 2: 517-524. DOI: 10.1016/j.sigpro.2012.08.022 